\title{
Gauss's Law: Re-Examination and Its Consequences
}

\author{
Narahari V. Joshi \\ Faculty of Science, University of Los Andes, Merida, Venezuela \\ Email: Joshi@ula.ve
}

Received 19 October 2015; accepted 14 November 2015; published 17 November 2015

Copyright (C) 2015 by author and Scientific Research Publishing Inc.

This work is licensed under the Creative Commons Attribution International License (CC BY). http://creativecommons.org/licenses/by/4.0/

(c) (i) Open Access

\begin{abstract}
Gauss's law is modified to take into account the absorption of the electric field by vacuum. It has important consequences as the absorbed energy becomes a part of the vacuum energy and excites the energy of strings in a form of quantum harmonic oscillators. Thus, vacuum becomes an excitable medium and this helps to understand the basic mechanisms for the conservation of the charge of the electron, electrostatic attraction or repulsion and the energy associated with vacuum.
\end{abstract}

\section{Keywords}

\section{Gauss Law, Vacuum Energy, String Theory}

\section{Introduction}

One of the most fundamental and widely used principles in electricity and magnetism is given by Gauss's law. It deals with the flow of energy associated with an electrical field $E$. If there is no charge in the enclosed area in the vacuum, then the incoming electric flux is equal to the outgoing electric flux; and if there is a charge inside the closed surface then the flow is given by [1]

$$
Q / e_{0}=\nabla \cdot E .
$$

Here $Q$ is the total charge inside the closed surface in the vacuum and $e_{0}$ is the dielectric constant of vacuum. This sounds well if the absorption of the energy within the vacuum associated with the electric field is not considered.

It is known that the flux of $E$ coming through the closed surface is equal to the outgoing flux. This turns out due to the fact that the relation between the intensity of the electric field, at a distance $r$ from the charge, is proportional to $1 / r^{2}$ and the area of the sphere is proportional to $r^{2}$ and hence the product is independent of $r$ [1]. So, the input flux appears to be equal to the output flux. 


\section{Analysis}

It is, therefore, necessary to look at the flow of the electric energy $E$ through the volume of cones of radius $R_{1}$ and $R_{2}$ originated at the point charge $Q$ (Figure 1). The energy density (energy/area) passing through the volumes $R_{1} \mathrm{~d} \theta R_{1} \mathrm{~d} \Phi \mathrm{d} r$ and $R_{2} \mathrm{~d} \theta R_{2} \mathrm{~d} \Phi \mathrm{d} r$ which enclose the cone needs to be examined. The energy passing through both volumes in a fixed time $\mathrm{d} t$ should be equal if there is no absorption of energy in the vacuum. The distance travelled by the energy in time $\mathrm{d} t$ is $c \mathrm{~d} t=\mathrm{d} r$; where $c$ is the velocity of light. Therefore, the same thickness of the shell $\mathrm{d} r$ is used in both cases to estimate the flow of energy in a fix time $\mathrm{d} t$.

The energy associated with the electric field per unit volume is given by [1]

$$
U=1 / 2\left(e_{0} E^{2}\right)
$$

$E$ at any point on the surface is given by

$$
E=\frac{1}{4 \pi \varepsilon_{0}} \frac{Q}{r^{2}}
$$

where $r$ is the distance measured from charge $Q$. Therefore, the energy per unit volume is given by

$$
U=\frac{1}{32 \pi^{2} \varepsilon_{0}} \frac{Q^{2}}{r^{4}} .
$$

The energy passing through the volume of the cone $\left(R_{1} \mathrm{~d} \theta R_{1} \mathrm{~d} \Phi \mathrm{d} r\right)$ located at a distance $R_{1}$ from charge $Q$ with thickness $\mathrm{d} r$ is given by

$$
U=\mathrm{d} \theta \mathrm{d} \Phi \frac{1}{32 \pi^{2} \varepsilon_{0}} Q^{2} \int_{R_{1}}^{R_{1}+\mathrm{d} r} \frac{1}{r^{4}} r^{2} \mathrm{~d} r
$$

where $\mathrm{d} \theta$ and $\mathrm{d} \Phi$ are angles subtended by the surface at the point charge $Q$.

$$
\begin{aligned}
& U=\mathrm{d} \theta \mathrm{d} \Phi \frac{1}{32 \pi^{2} \varepsilon_{0}} Q^{2} \int_{R_{1}}^{R_{1}+\mathrm{d} r} \frac{1}{r^{2}} \mathrm{~d} r \\
& U=\mathrm{d} \theta \mathrm{d} \Phi \frac{1}{32 \pi^{2} \varepsilon_{0}} Q^{2} \frac{\mathrm{d} r}{R_{1}\left(R_{1}+\mathrm{d} r\right)} .
\end{aligned}
$$

The energy passing out through the volume of the cone located at $R_{2} \mathrm{~d} \theta R_{2} \mathrm{~d} \Phi \mathrm{d} r$ is

$$
U=\mathrm{d} \theta \mathrm{d} \Phi \frac{1}{32 \pi^{2} \varepsilon_{0}} Q^{2} \frac{\mathrm{d} r}{R_{2}\left(R_{2}+\mathrm{d} r\right)} .
$$

As $R_{2}>R_{1}$, (Figure 1 ) the input flow is higher than the output flow and this shows undoubtfully that the energy is absorbed by the medium. If there is no absorption of the field in the vacuum, exactly the same energy is expected to pass through the surface of the second cone. But, it is not the same. The magnitude of Equation (7)

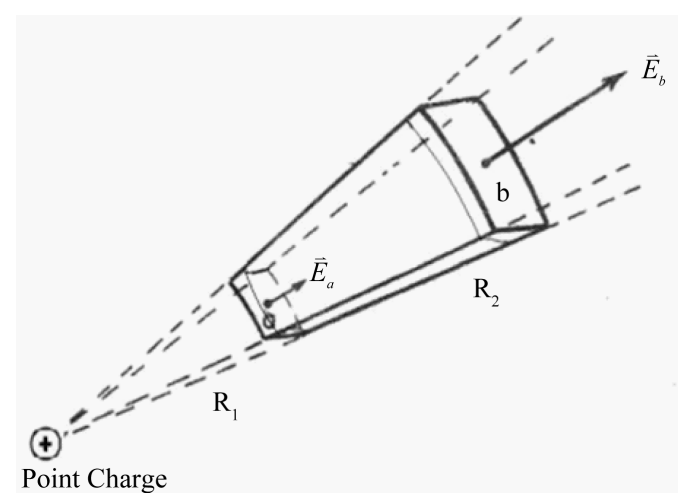

Figure 1. The flow of electric energy $E_{a}$ entering through volume $a$ and $E_{b}$ going out through volume $b$. 
is more than Equation (8). This indeed confirms that the electric field is absorbed by the vacuum. Therefore Equation (1) needs to be modified

$$
Q / e_{0}=\nabla \cdot E+\delta E
$$

where $\delta E$ is the energy absorbed by the vacuum which makes it an excitable medium for many useful purposes. Moreover, it also explains partially how vacuum acquires energy convertible to electric energy. Equation (9) clearly indicates that the electric field is not something which only has vector properties and flow in the vacuum but also it has capacity to interact with the medium and it is absorbed partially without making any measurable change in the system. This feature provides significant information about both the medium as well as the field. The details will be discussed later.

The above approach helps to solve the long standing puzzle related with the emission of electric field by the electron (or the charge particle). Let us consider that the electron emits energy in the form of electric field for time $t$. The electric field is created within a radius of distance $c t$. After time $\mathrm{d} t$, the electric field is observed within a radius of distance $c(t+\mathrm{d} t)$ without altering the intensity of the electric field within a radius $c t$. This means that an additional energy is created within the volume enclosed in circular layer $[c(t+\mathrm{d} t)-c t]$. Moreover, the space (or the vacuum) is being filled at every instant with electric field (energy) and during the process the mass of the electron is constant. So the principle of conservation of energy is clearly violated. Surprisingly, this significant aspect has not received any attention. This critical situation is overcome with the help of Equation (9) which takes into account the absorption of electric field by vacuum.

\section{Discussion}

The presence of vibrational energy in the vacuum is also confirmed by Casimir effect [2] [3] which shows the presence of zero point energy of the quantized field.

The absorption of the electric field in the vacuum is a direct process and provides information about the nature and the interaction of the electric field. The energy of the self excited vibrational modes associated with the electric field becomes the part of the vibrational energy of the strings which forms the system of the compact liquid as considered earlier [4]-[8]. The absorbed electric energy excites the harmonic oscillations associated with strings to the higher level and it becomes the part of the buffer energy of the strings or the vacuum which is used in the wave propagation process also.

The electron or the charge particle is emitting energy forever and its mass is constant. This indicates that the charge particle is receiving energy by a feedback mechanism from the vacuum as discussed earlier by Joshi [4]. The absorption process discussed above throws light on the details of the feedback mechanism. Let us examine it in a close region near the electron (or charge particle) and its consequences. As the magnitudes of $R_{1}$ and $R_{2}$ in Equations (7) and (8) are very small, the absorption of the electric field is significantly high in a close proximity of the electron. This indicates that the amplitude of the frequency corresponding to the electric field is very high.

The close relationship between high amplitude of the wave originated by wake oscillator and feed back mechanism is given by Van der Pol equation which is generally used in compressed fluids [4] [9] and it is given by [9]

$$
\frac{\mathrm{d}^{2} X}{\mathrm{~d} t^{2}}-D\left(1-X^{2}\right) \frac{\mathrm{d} X}{\mathrm{~d} t}+X=0
$$

where $X$ is the amplitude and $D$ is the damping coefficient. Van der Pol equation [9] is one of the few equations which represent feedback in oscillatory mechanism. Negative damping coefficient indicates that the amplitude of vibrations increases once they have started and feedback becomes a reality. The details of this non linear system applied for fluid is discussed extensively earlier [4] [9]. As the intensity or the amplitude of the vibration is increased due to the absorption of the field in the region very close to the electron (or charge particle), the negative damping and the feedback mechanism are the natural consequences of the absorption process.

To explain precisely the mechanism of absorption of electric energy is rather difficult as the nature of the electric field itself is not well understood on the basis of classical electrodynamics. It is clear and experimentally confirmed by several techniques that the field which we are dealing has characteristics of vector. It has magnitude, direction and it follows the laws of vector analysis [1]. The electric field is something which has a quality of motion which permits to introduce the concept of input or output flow of energy in a specific form. Some times it is considered as an abstract concept to explain the lines of forces, the stress created in a specific region 
or the flow of energy which participates in the interaction process with other particles or any other field.

Recently, it has been confirmed that the space is filled with strings in a compact form of liquid [5] [6] and several electro-magnetic properties have been explained with the help of vibrating strings without considering $D$-branes or any other extra dimension. It is also suggested that strings are vibrating units and have the property of self excitation. With the presence of charge, self excited vibrational modes are generated and propagated in the space. The vibrational energy of strings can be associated with the electric energy as it follows vector properties and propagates like an electrical field. This is the reason why there is no a stable equilibrium point in any electrostatic field (without considering the origin) except on the top of the charge. This aspect has been explained by a feedback mechanism of vibrating strings very close to the charge particle [4]. Moreover, it is found that the smaller the dimensions of the vibrating unit (like in the present case) the system becomes flexible, continuous and one dimensional like a thread (or line) which carries energy (or force) and reacts with the other systems of particle or fields [10]. This is the origin for the "field lines" and helps to understand the concept "lines of force” in dealing several aspects of the electric field.

According to the Quantum Field Theory (Q.F.T.), under specific conditions, the field can be converted into a particle (or vice versa). This is a consequence of the nature of the field which is strongly associated with self excited vibrations of the organized strings. According to Q.F.T. the particle is considered as an excited state of the underlying physical field and hence interacts and is considered as a "Field Quanta” [11]. The absorption of electric field in the vacuum is a direct process and throws light on the nature and interaction of the electric field.

Self excited vibrations form a system of coupled quantum harmonic oscillators and carries kinetic, potential and buffer energies. This makes it more versatile and this is confirmed by several experiments. One of the most important accomplishments of the present approach is that it helps to understand the conversion of fields into a particle (or vice versa) which plays a significant role in quantum field theory. Using this approach, Maxwell's equations and wave propagation have been reexamined successfully and concluded that space becomes an excitable medium with the presence of strings [7].

Equation (9) shows that vacuum absorbs energy and it is circulated in various forms. A recent investigation reveals that the mechanisms of repulsion or attraction of two electric charges is based on the potential energy of strings in the space [8]. This means that the absorbed energy can be converted into potential energy of vibrating strings. Such studies clearly indicate that vacuum has energy associated with vibrating strings and can be converted into the electro-magnetic form and this is widely discussed [12] and some patents have been registered to convert vacuum energy in electric energy [13].

\section{Conclusion}

Gauss law needs to be modified to take into account the absorption of the electric field by strings in a compact form of liquid. The absorbed energy is stored as buffer energy and it is also used for electrostatic attraction and repulsion processes. This also plays a crucial role in wave propagation of energy and the conversion of energy from vacuum to electrical. A direct detection of the presence of strings in the form of a compact liquid is not possible because of their very small dimensions, unknown magnitudes of strain and other properties related with vibrations. Their presence can be confirmed only by indirect and consistent methods. The conclusion of the present work is that vacuum in the form of a compact, incompressible dry liquid of strings absorbs electromagnetic energy and it is used for feedback and storage purposes.

\section{References}

[1] Feynman, R.P., Leighton, R.B.M. and Sands, M. (1969) The Feynman Lectures on Physics. Vol. 2, Addision-Wesley, Boston.

[2] Milonni, P. (1994) The Quantum Vacuum: An Introduction to Quantum Electro Dynamics. Academic Press, San Diego.

[3] Maclay, J. (2000) Physical Review A, 61, Article ID: 052110. http://dx.doi.org/10.1103/PhysRevA.61.052110

[4] Joshi, N.V. (2013) Physics Essays, 36, 61-67. http://dx.doi.org/10.4006/0836-1398-26.1.61

[5] Joshi, N.V. (2014) World Journal of Mechanics, 4, 309-314.

[6] Joshi, N.V. (2014) World Journal of Mechanics, 4, 247-250.

[7] Joshi, N.V. (2015) Journal of Modern Physics, 6, 921-926. http://dx.doi.org/10.4236/jmp.2015.67096 
[8] Joshi, N.V. (2013) World Journal of Mechanics, 3, 307-309.

[9] Wiggins, S. (1990) Introduction to Nonlinear Dynamical System and Chaos. Springer Verlag, New York. http://dx.doi.org/10.1007/978-1-4757-4067-7

[10] Henely, E.M. and Thirring, W. (1962) Elementary Quantum Field Theory. McGraw-Hill, London.

[11] Tong, D. (2015) Lectures on Quantum Field Theory. Cambridge University, UK.

[12] Bearden, T.E. (2004) Energy from the Vacuum. Cheniere Press, Santa Barbara.

[13] Haisch, B. and Moddel, G. (2008) Quantum Vacuum Energy Extraction. Patent No. 7379286, USA. 